\title{
RNA Folding Algorithms with G-Quadruplexes
}

\author{
Ronny Lorenz ${ }^{1}$, Stephan H. Bernhart ${ }^{2}$, Fabian Externbrink ${ }^{2}$, Jing Qin ${ }^{3}$, \\ Christian Höner zu Siederdissen ${ }^{1}$, Fabian Amman ${ }^{1}$, Ivo L. Hofacker ${ }^{1,4}$, and \\ Peter F. Stadler $2,1,3,4,5,6$ \\ 1 Dept. Theoretical Chemistry, Univ. Vienna, Währingerstr. 17, Wien, Austria \\ 2 Dept. Computer Science, and Interdisciplinary Center for Bioinformatics, Univ. \\ Leipzig, Härtelstr. 16-18, Leipzig, Germany \\ 3 MPI Mathematics in the Sciences, Inselstr. 22, Leipzig, Germany \\ 4 RTH, Univ. Copenhagen, Grønnegårdsvej 3, Frederiksberg C, Denmark \\ ${ }^{5}$ FHI Cell Therapy and Immunology, Perlickstr. 1, Leipzig, Germany \\ ${ }^{6}$ Santa Fe Institute, 1399 Hyde Park Rd., Santa Fe, USA
}

\begin{abstract}
G-quadruplexes are abundant locally stable structural elements in nucleic acids. The combinatorial theory of RNA structures and the dynamic programming algorithms for RNA secondary structure prediction are extended here to incorporate G-quadruplexes using a simple but plausible energy model. With preliminary energy parameters we find that the overwhelming majority of putative quadruplex-forming sequences in the human genome are likely to fold into canonical secondary structures instead.
\end{abstract}

Key words: Dynamic programming, RNA folding, ViennaRNA Package

\section{Introduction}

Guanosine-rich nucleic acid sequences readily fold into four-stranded structures known as G-quadruplexes. DNA quadruplexes are, for instance, an important component of human telomeres [1], they appear to be strongly overrepresented in the promoter regions of diverse organisms, and they can associate with a variety of small molecule ligands, see $[2,3]$ for recent reviews. SNPs in G-quadruplexes, finally, have been implicated as a source variation of gene expression levels [4]. RNA quadruplexes have also been implicated in regulatory functions. Conserved G-quadruplex structures within the 5'-UTR of the human TRF2 mRNA [5] and eukaryotic MT3 matrix metalloproteinases, for example, repress translation [6]. Another well-studied example is the interaction of the RGG box domain fragile $\mathrm{X}$ mental retardation protein (FMRP) to a G-quartet-forming region in the human semaphorin 3F (S3F) mRNA $[7,8]$. A recent review of G-quadruplexbased translation regulation is [9]. A functional RNA G-quadruplex in the 3' UTR was recently described as a translational repressor of the proto-oncogene PIM1 [10]. A mechanistic study of this effect, which seems to be widely used in the cell $[11,12]$ can be found e.g. in [13]. Most recently, G-quadruplexes were also reported in several long non-coding RNAs [14]. G-quadruplexes are potentially 

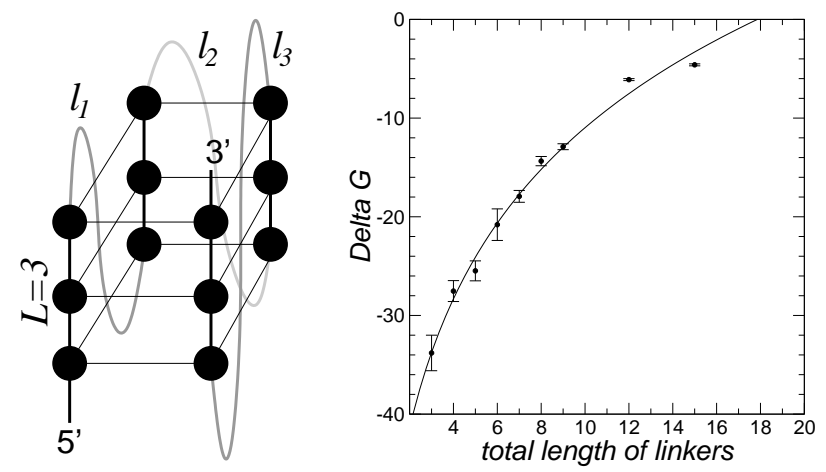

Fig. 1. RNA quadruplexes form parallel arrangements with $L=$ $2 \ldots 5$ layers. Folding energies for $L=3$ depend mostly on the total length $\ell$ of the linker sequences: the data from ref. [16] fit well to an energy model of the form $\Delta G=a+b \ln \ell$ (solid line).

of functional importance in the 100 to $9000 \mathrm{nt}$ G-rich telomeric repeat-containing RNAs (TERRAs) [15].

Quadruplex structures consist of stacked associations of G-quartets, i.e., planar assemblies of four Hoogsteen-bonded guanines. As in the case of base pairing, the stability of quadruplexes is derived from $\pi$-orbital interactions among stacked quartets. The centrally located cations that are coordinated by the quartets also have a major influence on the stability of quadruplex structures.

DNA quadruplexes are structurally heterogeneous: depending on the glycosidic bond angles there are 16 possible structures and further combinatorial complexity is introduced by the relative orientations of the backbone along the four edges of the stack [17]. RNA quadruplexes, in contrast, appear to be structurally monomorphic forming parallel-stranded conformations (Fig. 1, left) independently of surrounding conditions, i.e., different cations and RNA concentration [18]. Here, we restrict ourselves to the simpler case of RNA quadruplexes.

Bioinformatically, G-quadruplex structures have been investigated mostly as genomic sequence motifs. The G4P Calculator searches for four adjacent runs of at least three Gs. With its help a correlation of putative quadruplex forming sequences and certain functional classes of genes was detected [19]. Similarly, quadparser [20] recognizes the pattern (1) below. It was used e.g. in [21] to demonstrate the enrichment of quadruplexes in transcriptional regulatory regions. A substantial conservation of such sequence patterns in mammalian promoter regions is reported in [22]. The web service QGRS Mapper uses a similar pattern and implements a heuristic scoring system [23], see also [24] for a review. A Bayesian prediction framework based on Gaussian process regression was recently introduced to predict melting temperatures of quadruplex sequences [25].

The formation of RNA quadruplexes necessarily competes with the formation of canonical secondary structures. Hence they cannot be fully understood in isolation. In this contribution we therefore investigate how G-quadruplex structures can be incorporated into RNA secondary structure prediction algorithms. 


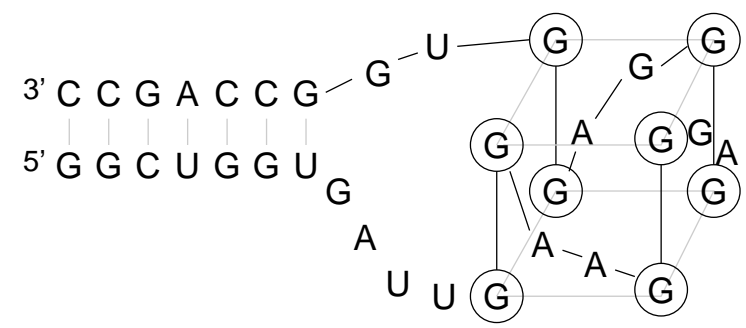

Fig. 2. Structure of the Gquadruplex in a hairpin of human semaphorin 3F RNA that binds the RGG box domain of fragile $\mathrm{X}$ mental retardation protein (FMRP). Redrawn based on [7].

\section{$2 \quad$ Energy Model for RNA Quadruplexes}

Thermodynamic parameters for RNA quadruplexes can be derived from measurements of UV absorption as a function of temperature [26], analogous to melting curves of secondary structures. While the stability of DNA G-quadruplexes strongly depends on the arrangement of loops [27, 28] this does not appear to be the case for RNA. RNA not only forms mostly parallel-stranded stacks for Gquartets but their stability also exhibits a rather simple dependence of the loop length [16]. In further contrast to DNA [29], they appear to be less dependent on the nucleotide sequence itself.

A G-quadruplex with $2 \leq L \leq 5$ stacked G-quartets and three linkers of length $l_{1}, l_{2}, l_{3} \geq 1$ has the form

$$
G_{L} N_{l_{1}} G_{L} N_{l_{2}} G_{L} N_{l_{3}} G_{L}
$$

It is commonly assumed that $1 \leq l_{i} \leq 7$ [25], although in vitro data for DNA suggest that longer linkers are possible [30]. For $L=2$, the existence of quadruplexes with $1 \leq \ell_{i} \leq 2$ was reported [31]. For $L=3$ detailed thermodynamic data are available only for the 27 cases $1 \leq l_{1}, l_{2}, l_{3} \leq 3$ and for some longer symmetric linkers $l_{1}=l_{2}=l_{3}[16]$, see Figure $1 \mathrm{~b}$. To our knowledge, no comprehensive data are available for $L \geq 4$. It appears reasonable to assume that the stacking energies are additive. The energetic effect of the linkers appears to be well described in terms of the total linker length $\ell$ [16]. As shown in Figure 1b the free energy depends approximately logarithmically on $\ell$. In this contribution we are mostly concerned with the algorithmic issues of including G-quadruplexes into thermodynamic folding programs. In particular we ignore here the strong dependence of quadruplex stability on the potassium concentration, see e.g. [32]. We thus resort to the simplified energy function

$$
E[L, \ell]=a(L-1) g_{0}+b \ln (\ell-2)
$$

with parameters $a=-18 \mathrm{kcal} / \mathrm{mol}$ and $b=12 \mathrm{kcal} / \mathrm{mol}$ if the pattern (1) is matched, and $E=\infty$ otherwise.

G-quadruplex structures can be located within loops of more complex secondary structures. Fig. 2, for instance, shows the $L=2, l_{1}=l_{2}=l_{3}=2$ quadruplex in a hairpin of the semaphorin 3F RNA [7]. It seems natural to treat G-quadruplexes inside multiloops similar to their branching helices: each 
unpaired base incurs a penalty $a$ and each G-quadruplex within a loop is associated with an additional "loop strain" $b$. For the interior-loop case of Fig. 2, only stabilizing mismatch contributions of the enclosing pair and a penalty for the stretches of unpaired bases are used. Sterical considerations for this case suggest that a G-quadruplex is flanked by a stretch of at least three unpaired nucleotides or has at least one unpaired nucleotide on either side.

\section{Combinatorics of Structures with Quadruplexes}

RNA secondary structures consist of mutually non-crossing base pairs and unpaired positions. Thus they can be represented as strings composed of matching parentheses (base pairs) and dots. This "dot-parenthesis" notation is used by the ViennaRNA Package [33]. G-quadruplexes constitute an extra type of structural element. The semaphorin hairpin, Fig. 2, can therefore be written as

GGCUGGUGAUUGGAAGGGAGGGAGGUGGCCAGCC

using the symbol + to mark the bases involved in G-quartets. This string representation uniquely identifies all G-quartets since the first run of + symbols determines $L$ for the 5 '-most quadruplex, thus determining the next three G-stacks which are separated by at least one '.' and must have the same length. It follows immediately that the number of secondary structures with G-quadruplexes is still smaller than $4^{n}$, an observation that is important for the evolvability of RNAs [34]. In order to get a tighter bound on the number of structures we use here, for the sake of presentation, a simplified model in which we omit the restrictions of a minimal size of a hairpin loop and allow quadruplexes with any value of $L \geq 2$ and $l_{i} \geq 1$.

Let $\mathbf{g}_{n}$ denote the number of secondary structures with $\mathrm{G}$ quadruplexes on a sequence of length $n$. The corresponding generating function is $\mathbf{G}(x)=$ $\sum_{n \geq 0} \mathbf{g}_{n} x^{n}$. Similarly, let $\mathbf{q}_{n}$ be the number of quadruplexes on length $n$. As derived in the supplement, its generating function is $\mathbf{Q}(x)=\sum_{n>0} \mathbf{q}_{n} x^{n}=$ $x^{11}(1-x)^{-3}\left(1-x^{4}\right)^{-1}$. The basic idea is now to consider a structure consisting of $b$ base pairs, $u$ unpaired bases and $k$ quadruplexes. Then there are $\left(\begin{array}{c}2 b+k \\ k\end{array}\right)$ ways

to insert $k$ quadruplexes into each of the $C_{b}=\frac{1}{b+1}\left(\begin{array}{c}2 b \\ b\end{array}\right)$ possible arrangements of $b$ matching pairs of parentheses. Into each of these arrangements we can insert $u$ unpaired bases in $\left(\begin{array}{c}2 b+k+u \\ u\end{array}\right)$ different ways. Thus we have

$$
\begin{aligned}
\mathbf{G}(x) & =\sum_{k} \sum_{b} \sum_{u} \frac{1}{b+1}\left(\begin{array}{c}
2 b \\
b
\end{array}\right)\left(\begin{array}{c}
2 b+k \\
k
\end{array}\right)\left(\begin{array}{c}
2 b+k+u \\
u
\end{array}\right) x^{2 b+u} \mathbf{Q}(x)^{k} \\
& =\frac{2}{1-x-\mathbf{Q}(x)+\sqrt{(1-3 x-\mathbf{Q}(x))(1+x-\mathbf{Q}(x)))}}
\end{aligned}
$$

Following [35] we find that the coefficients of $\mathbf{G}(x)$ are asymptotically given by $\mathrm{g}_{n} \sim k_{0} n^{-3 / 2} \gamma^{n}$, where $k_{0}$ is a positive constant and $\gamma \approx 3.00005$. A more detailed model accounting for minimal stack and loop lengths is analyzed in the 


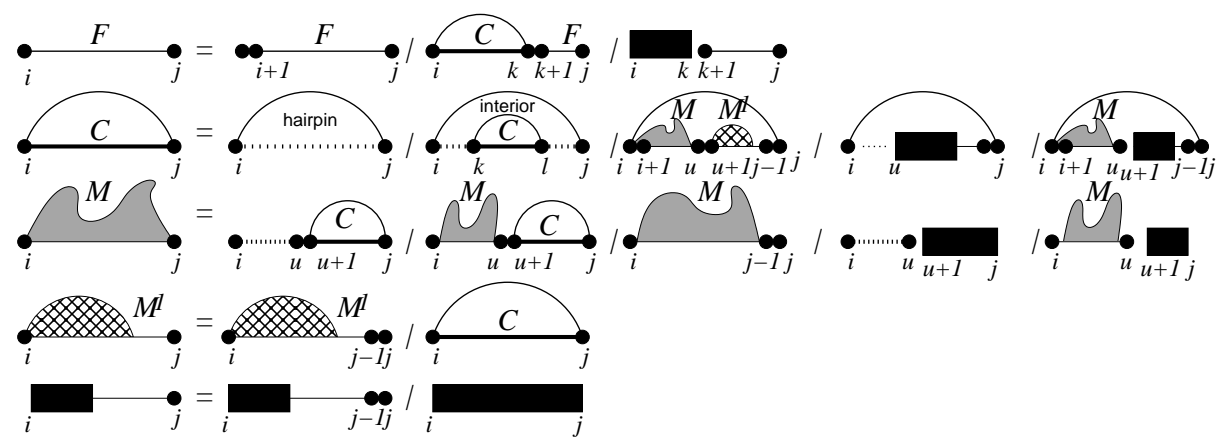

Fig. 3. Extension of recursions of the ViennaRNA Package to accomodate Gquadruplexes. This grammar treats G-quadruplexes with multi-loop like energies also in an interior-loop-like context.

Supplemental Material. It yields $\gamma \approx 2.2903$ if isolated base pairs are allowed, and $\gamma \approx 1.8643$ for canonical secondary structures.

\section{RNA Folding Algorithms}

Energy Minimization. Dynamic programming algorithms for secondary structure prediction are based on a simple recursive decomposition: any feasible structure on the interval $[i, j]$ has the first base either unpaired or paired with a position $k$ satisfying $i<k \leq j$. The condition that base pairs do not cross implies that the intervals $[i+1, k-1]$ and $[k+1, j]$ form self-contained structures whose energies can be evaluated independent of each other. In conjunction with the standard energy model [36], which distinguishes hairpin loops, interior loops (including stacked base pairs), and multi-loops, this leads to the recursions diagrammatically represented in Fig. 3 (ignoring the cases involving black blocks). This algorithmic approach was pioneered e.g. in $[37,38]$ and is also used in the ViennaRNA Package [33].

G-quadruplexes form closed structural elements on well-defined sequence intervals. Thus they can be treated just like substructures enclosed by a base pair, so that the additional ingredients in the folding algorithms are the energies $G_{i j}$ (free energy of the most stable quadruplex so that the pattern (1) matches exactly the interval $[i, j]$ ) and the partition functions $Z_{i j}^{G}$ (defined as the sum of the Boltzmann factors of all distinct quadruplexes on the interval $[i, j])$. As a consequence of (1) we have $G_{i j}<\infty$ and $Z_{i j}^{G}>0$ only if $|j-i|<4 L_{\max }+\ell_{\max }$. All possible quadruplexes on the interval $[i, j]$ can be determined and evaluated in $\mathcal{O}\left(L_{\max }^{2} \ell_{\max }^{2}\right)$ time so that these arrays can be precomputed in $\mathcal{O}\left(n\left(L_{\max }+\ell_{\max }\right) L_{\max }^{2} \ell_{\max }^{2}\right)$, i.e., in linear time.

The standard recursions for RNA secondary structure prediction can now be extended by extra terms for quadruplexes, see Fig. 3. The simplest strategy 
would be to add G-quadruplexes as an additional type of base-pair enclosed structures. This would amount to using standard interior loop parameters also for cases such as Fig. 2. Hence we use the somewhat more elaborate grammar of Fig. 3, which introduces the quadruplexes in the form of additional cases into the multi-loop decomposition. An advantage of this method is that one can use different parameter values to penalize the inclusion of quadruplexes and helical components into a multiloop. Clearly the grammar is still unambiguous, i.e., every structure has an unique parse. Thus it can be used directly to compute partition functions.

Base Pairing Probabilities. A straightforward generalization of McCaskill's algorithm can be used to compute the probabilities $P_{i j}$ of all possible base pairs $(i, j)$. The probability $P_{i j}^{G}$ of finding a G-quadruplex delimited by positions $i$ and $j$ then can be written as

$$
P_{i j}^{G}=\frac{Z_{1, i-1} Z_{i j}^{G} Z_{j+1, n}}{Z}+\sum_{\substack{k<i-1 \\ l>j+1}} P_{k l} \mathbb{P}\{\text { quadruplex }[i, j] \mid(k, l)\}
$$

The conditional probabilities $\mathbb{P}\{\ldots\}$ in turn are composed of the four individual cases depending on the placement of the components of the generalized multiloop enclosed by $(k, l)$ relative to the interval $[i, j]$ :

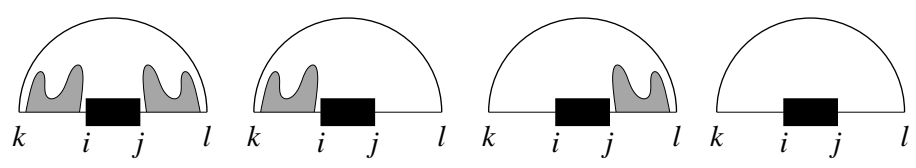

This decomposition translates to the recursion $\mathbb{P}\{$ quadruplex $[i, j] \mid(k, l)\}=$

$$
\frac{Z_{k+1, i-1}^{M} Z_{i j}^{G} Z_{j+1, l-1}^{M}}{Z_{k l}^{B}}+\frac{Z_{k+1, i-1}^{M} Z_{i j}^{G} \hat{b}^{l-j-1}}{Z_{k l}^{B}}+\frac{\hat{b}^{i-k-1} Z_{i j}^{G} Z_{j+1, l-1}^{M}}{Z_{k l}^{B}}+\frac{\hat{b}^{i-k-1} Z_{i j}^{G} \hat{b}^{l-j-1}}{Z_{k l}^{B}}
$$

where $\hat{b}=\exp (-b / R T)$. From the $P_{i j}^{G}$ it is straightforward to compute the probability of a particular quadruplex as

$$
p\left(\left[i, L, l_{1}, l_{2}, j\right]\right)=\frac{\exp (-E[L, \ell])}{Z_{i j}^{G}} P_{i j}^{G}
$$

where $l_{3}=j-i+1-4 L-l_{1}-l_{2}$. Summing up the probabilities of all quadruplexes that contain a particular contact $i^{\prime}: j^{\prime}$ of two guanosines in a layer finally yield the probability of the G:G contact $i^{\prime}: j^{\prime}$.

Fig. 4 shows an example of the graphical output of RNAfold. In the minimum energy case we use a very simple modification of the standard layout [39] treating each quadruplex like a local hairpin structure, explicitly indicating the G-G pairs. Quadruplexes are shown in addition to the individual G-G pairs as shaded triangles in the base pair probability dot plots. From the base pairing probabilities we also compute MEA [40] and centroid structures. 


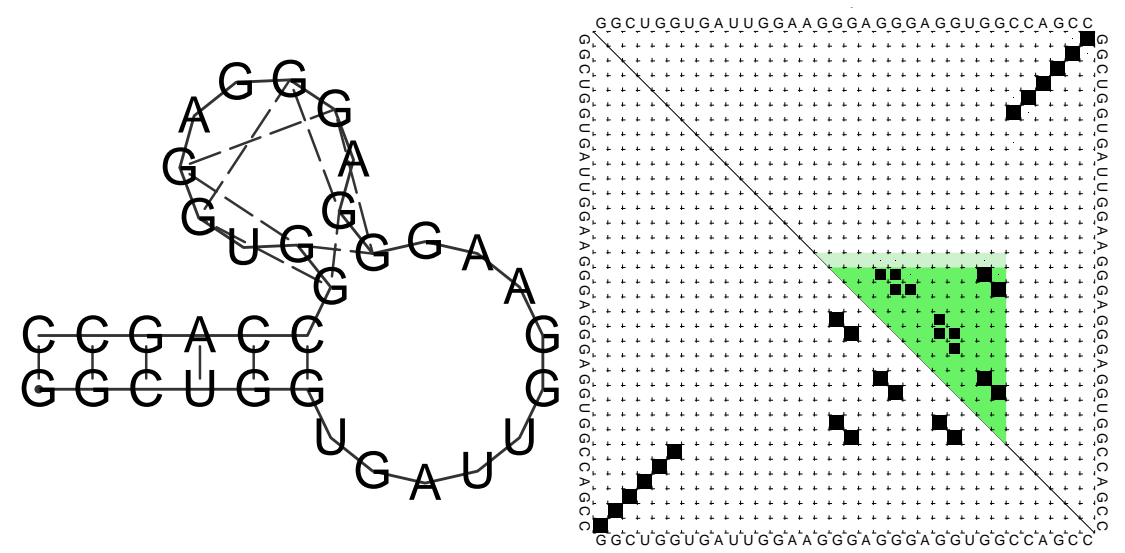

Fig. 4. Representation of minimum free energy structure (1.h.s.) and base pairing probability matrix (r.h.s.) of the semaphorin hairpin (see Fig. 2) respectively.

By definition the centroid structure $X$ minimizes the expected base pair distance to the other structures within the Boltzmann-weighted ensemble. In the absence of G-quadruplexes $X$ consists of all base pairs $(i, j)$ with $p_{i j}>1 / 2$. A certain ambiguity arises depending on whether $X$ is interpreted as a list of base pairs that may contain incomplete quadruplexes, or whether quadruplexes are treated as units. Here, we insert a quadruplex if $P_{i j}^{G}>0.5$, and represent it by the most stable quadruplex with endpoints $i$ and $j$. The same representation is used for MEA structures where we extend the maximized expected accuracy to $\mathrm{EA}=\sum_{(i, j) \in S} 2 \gamma\left(P_{i, j}+P_{i j}^{G}\right)+\sum_{i} P_{i}^{u}$ with $P_{i}^{u}=1-\sum_{j} P_{i j}-\sum_{k \leq i \leq l} P_{k l}^{G}$, accordingly.

Consensus Structures can be readily obtained for a given multiple sequence alignment. The idea is to apply the dynamic programming recursions to alignment columns. The energy contributions are determined as the average of the corresponding contributions to the individual sequences [42]. In addition small contributions are added to favor pairs of columns with consistent (e.g. $\mathrm{GC} \rightarrow \mathrm{GU}$ ) and compensatory mutations $(\mathrm{AU} \rightarrow \mathrm{GC}$ ) since these provide direct evidence for selection acting to preserve base pairing. Similarly, penalties are added if one or a few sequences cannot form a base pair. We refer to [43] for details of the scoring model implemented in RNAalifold. Here, we extend it by a simple system of penalties for mutations that disrupt quadruplexes. Non-G nucleotides incur an energy $E^{\prime}$ in the outer layers of the quadruplex and $2 E^{\prime}$ in the inner layers as they affect one or two stacking interactions, respectively. An example of a consensus structure prediction is shown in Fig. 5.

Implementation Details. The implementation of G-quadruplex folding in RNAfold and RNAalifold essentially follows the extended grammar shown in Fig. 3, distinguishing the energy contribution of unpaired bases in the external loop from those enclosed by base pairs. The energies of all possible Gquadruplexes are pre-computed, storing the energy of the most stable quadruplex 

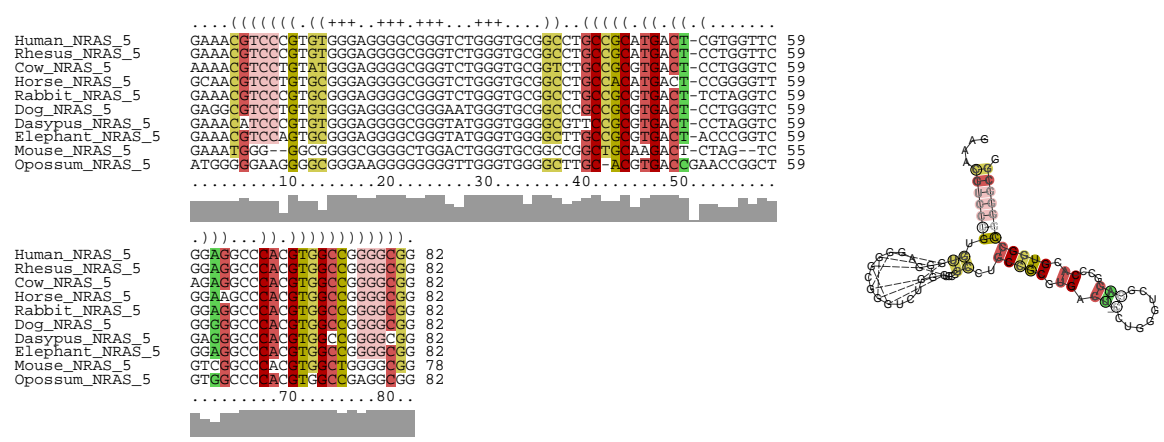

Fig. 5. Consensus structure of the 5'-most part of the 5'UTR of the NRAS mRNA, exhibiting a conserved G-quadruplex with $L=3$ that modulates translation of the NRAS proto-oncogene [41]. Colors indicate the number (red 1, ochre 2, green 3) of different types of basepairs in a pair of alignment columns, unsaturated colors indicate basepairs that cannot be formed by 1 or 2 sequences. Substitutions in stem regions are indicated by circles in the secondary structure drawing.

for each pair of endpoints in the triangular matrix $G$. As this matrix will be very sparse for most inputs, a sparse matrix optimization is possible, but not yet implemented. In the backtracing part we re-enumerate quadruplexes with given endpoints whenever necessary. Base pairing probabilities are computed as outlined above. Since there cannot be a conflict with canonical base pairs, we store $P_{i j}^{G}$ as part of the base pairing probability matrix. The probabilities of individual G-G contacts are computed by enumeration as a post-processing step. We also adapted the RNAeval and RNAplot programs so that sequence/structure pairs can be parsed and re-evaluated according to the extended grammar.

\section{Evaluation}

Runtime Performance. The runtime of RNAfold with the extended grammar of Fig. 3 was compared to the implementation of the standard model. For both, energy minimization and partition function, virtually no difference was observed. For short sequences of about $200 \mathrm{nt}$ the additional pre-processing steps incur a minor but negligible runtime overhead.

Occurrence and stability of G-quadruplexes in genomes. Sequence motifs of the form (1) that can in principle form quadruplex structures are very abundant in most genomes, see e.g. [19-21]. The number of putative quadruplexforming sequences is even slightly larger than expected from random sequences with the same mono- or dinucleotide distributions, Fig. 6 . The overwhelming majority of these quadruplex candidates, however, is unstable compared to canonical secondary structures that use some or all of Gs in canonical base pairs. We observe that less than $2 \%$ of the putative quadruplexes are thermodynamically stable. Interestingly, this effect is nearly independent of the number of layers $(L)$. This data is preliminary, since it is based on energy parameters that have 


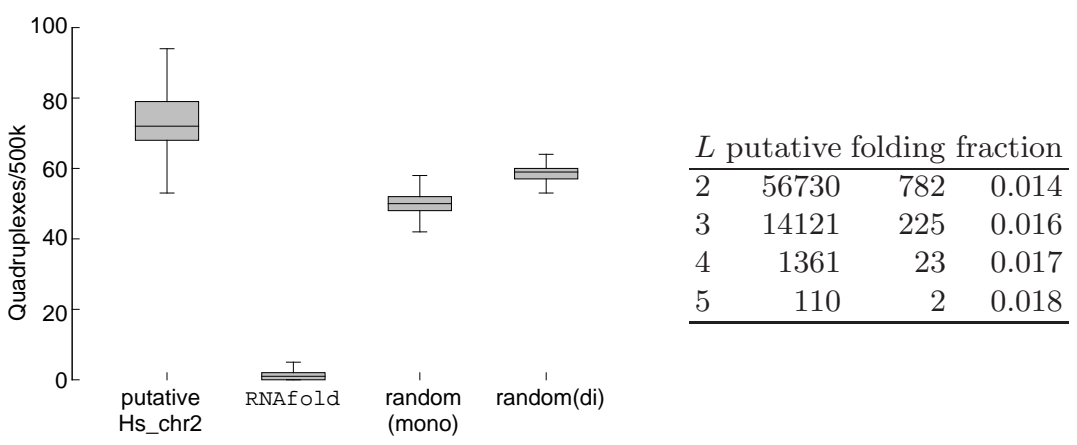

Fig. 6. Abundance and stability of putative G-quadruplexes. L.h.s.: Box plot showing the number of potential G-quadruplexes in human chromosome 2 within sliding windows of 500000 nucleotides. For comparison, the same information for a random sequence with the same mono- or di-nucleotide composition than chr. 2 is presented as well. Both, the mono- and di-nucleotide distribution have been generated from chromosome 2. RNAfold denotes the number of putative G-quadruplexes stable enough to occur in a predicted structure of 100 nucleotides up- and downstream of the putative G-quadruplex (with median=1, interquartile range=0-2). R.h.s.: fraction of stable quadruplexes as function of $L$. for human chromosome 2 .

been fitted on a rather limited amount of empirical evidence and do not include the important issues arising from the strong dependence of quadruplex stability of cation concentrations. Furthermore, it reflects the occurrance of putative G-quadruplexes on the human chromosome 2 only. More comprehensive and accurate parameters as well as a local folding algorithm that extends RNALfold [44] will be subject of a forthcoming study. Several experimentally know RNA quadruplexes are predicted by the current version, including the semphorin hairpin of Fig. 4 and the quadruplex in human telomerase RNA [45] discussed in the Supplemental Material.

\section{Discussion}

We have shown in this contribution that structural elements such as G-quadruplexes that correspond to uninterrupted sequence intervals can be included in a rather straightforward way into the standard dynamic programming recursions - provided a corresponding extension of the energy model can be devised. The G-quadruplex-aware programs are currently available as a separate branch (version number with the suffix "g") of the ViennaRNA Package using a very simple energy function for the quadruplexes that reproduces the few available experimental data at least semi-quantitatively. Following further optimization of the code the algorithmic extensions will be integrated in the main version of the package in the near future. The extensions in Fig. 3 can also be applied to local folding algorithms such as RNALfold and RNAplfold or the exhaustive 
enumeration of suboptimal structures in RNAsubopt. This is ongoing work, as is a comprehensive set of tools for genome-wide scans for putative G-quadruplexes.

It is less obvious how to handle quadruplexes in RNA-RNA interactions since our recursions consider local G-quadruplexes only. At least it is clear that they can be included in all those parts of the structure that are not involved in intermolecular contacts. Some quadruplex structures, however, are formed in trans. The binding of G-rich small RNAs to G-rich regions in reporter mRNAs leads to the formation of an intermolecular RNA G-quadruplex that in turn can inhibit translation in living cells [46]. One can use RNAup [47] to compute the probabilities $p^{(1)}$ and $p^{(2)}$ that the G-rich regions are unpaired. From these, we obtain the free energies $G^{(i)}=-R T \ln p^{(i)}$ to make the binding site accessible. It remains to compute the interaction energy itself.

The main problem for practical applications of quadruplex-aware RNA folding tools is our limited knowledge of the energy function in particular for $L \neq 3$ and for asymmetric linkers. Even with the crude energy function employed here it becomes clear that the overwhelming majority of putative genomic quadruplex sequences will fold into a canonical secondary structure rather than Gquadruplex structures.

Supplemental Material and Source Code are available from http://www. bioinf.uni-leipzig.de/publications/supplements/12-006 and www.tbi. univie.ac.at/ ronny/programs/.

Acknowledgements. This work was supported in part by the German Research Foundation (STA 850/7-2, under the auspicies of SPP-1258 "Sensory and Regulatory RNAs in Prokaryotes"), the Austrian GEN-AU projects "regulatory non coding RNA", "Bioinformatics Integration Network III" and the Austrian FWF project "SFB F43 RNA regulation of the transcriptome".

\section{References}

1. Paeschke, K., Simonsson, T., Postberg, J., Rhodes, D., Lipps, H.J.: Telomere endbinding proteins control the formation of G-quadruplex DNA structures in vivo. Nature Struct. Mol. Biol. 12 (2005) 847-854

2. Johnson, J.E., Smith, J.S., Kozak, M.L., Johnson, F.B.: In vivo veritas: using yeast to probe the biological functions of G-quadruplexes. Biochimie 90 (2008) $1250-1263$

3. Wong, H.M., Payet, L., Huppert, J.L.: Function and targeting of G-quadruplexes. Curr Opin Mol Ther. 11 (2009) 146-155

4. Baral, A., Kumar, P., Halder, R., Mani, P., Yadav, V.K., Singh, A., Das, S.K., Chowdhury, S.: Quadruplex-single nucleotide polymorphisms (Quad-SNP) influence gene expression difference among individuals. Nucleic Acids Res. (2012)

5. Gomez, D., Guédin, A., Mergny, J.L., Salles, B., Riou, J.F., Teulade-Fichou, M.P., Calsou, P.: A G-quadruplex structure within the 5'-UTR of TRF2 mRNA represses translation in human cells. Nucleic Acids Res. 38 (2010) 7187-7198

6. Morris, M.J., Basu, S.: An unusually stable G-quadruplex within the 5'-UTR of the MT3 matrix metalloproteinase mRNA represses translation in eukaryotic cells. Biochemistry 48 (2009) 5313-5319 
7. Menon, L., Mihailescu, M.R.: Interactions of the G quartet forming semaphorin 3F RNA with the RGG box domain of the fragile X protein family. Nucleic Acids Res. 35 (2007) 5379-5392

8. Bensaid, M., Melko, M., Bechara, E.G., Davidovic, L., Berretta, A., Catania, M.V., Gecz, J., Lalli, E., Bardoni, B.: FRAXE-associated mental retardation protein (FMR2) is an RNA-binding protein with high affinity for G-quartet RNA forming structure. Nucleic Acids Res. 37 (2009) 1269-1279

9. Bugaut, A., Balasubramanian, S.: 5'-UTR RNA G-quadruplexes: translation regulation and targeting. Nucleic Acids Res. (2012) doi: 10.1093/nar/gks068.

10. Arora, A., Suess, B.: An RNA G-quadruplex in the 3' UTR of the proto-oncogene PIM1 represses translation. RNA Biology 8 (2011) 802-805

11. Huppert, J.L., Bugaut, A., Kumari, S., Balasubramanian, S.: G-quadruplexes: the beginning and end of UTRs. Nucleic Acids Res. 36 (2008) 6260-6268

12. Beaudoin, J.D., Perreault, J.P.: 5'-UTR G-quadruplex structures acting as translational repressors. Nucleic Acids Res. 38 (2010) 7022-7036

13. Wieland, M., Hartig, J.S.: RNA quadruplex-based modulation of gene expression. Chem. Biol. 14 (2007) 757-763

14. Jayaraj, G.G., Pandey, S., Scaria, V., Maiti, S.: Potential G-quadruplexes in the human long non-coding transcriptome. RNA Biolog 9 (2012) 81-86

15. Luke, B., Lingner, J.: TERRA: telomeric repeat-containing RNA. EMBO J. 28 (2009) 2503-2510

16. Zhang, A.Y., Bugaut, A., Balasubramanian, S.: A sequence-independent analysis of the loop length dependence of intramolecular RNA G-quadruplex stability and topology. Biochemistry 50 (2011) 7251-7258

17. Webba da Silva, M.: Geometric formalism for DNA quadruplex folding. Chemistry $13(9738-9745) 2007$

18. Zhang, D.H., Zhi, G.Y.: Structure monomorphism of RNA G-quadruplex that is independent of surrounding condition. J. Biotechnol. 150 (2010) 6-10

19. Eddy, J., Maizels, N.: Gene function correlates with potential for G4 DNA formation in the human genome. Nucleic Acids Res. 34 (2006) 3887-3896

20. Huppert, J.L., Balasubramanian, S.: Prevalence of quadruplexes in the human genome. Nucleic Acids Res. 33 (2005) 2908-2916

21. Zhao, Y., Du, Z., Li, N.: Extensive selection for the enrichment of G4 DNA motifs in transcriptional regulatory regions of warm blooded animals. FEBS Letters $\mathbf{5 8 1}$ (2007) 1951-1956

22. Verma, A., Halder, K., Halder, R., Yadav, V.K., Rawal, P., Thakur, R.K., Mohd, F., Sharma, A., Chowdhury, S.: G-quadruplex DNA motifs as conserved cis-regulatory elements. J. Med. Chem. 51 (2008) 5641-5649

23. Kikin, O., D'Antonio, L., Bagga, P.S.: QGRS mapper: a web-based server for predicting G-quadruplexes in nucleotide sequences. Nucleic Acids Res. 34 (2006) W676-W682

24. Todd, A.K.: Bioinformatics approaches to quadruplex sequence location. Methods 43 (2007) 246-251

25. Stegle, O., Payet, L., Mergny, J.L., MacKay, D.J.C., Huppert, J.L.: Predicting and understanding the stability of G-quadruplexes. Bioinformatics 25 (2009) i374-i382

26. Mergny, J.L., Lacroix, L.: Uv melting of g-quadruplexes. Curr Protoc Nucleic Acid Chem. Unit 17.1. (2009)

27. Bugaut, A., Balasubramanian, S.: A sequence-independent study of the influence of short loop lengths on the stability and topology of intramolecular DNA Gquadruplexes. Biochemistry 47 (2008) 689-697 
28. Zhang, D.H., Fujimoto, T., Saxena, S., Yu, H.Q., Miyoshi, D., Sugimoto, N.: Monomorphic RNA G-quadruplex and polymorphic DNA G-quadruplex structures responding to cellular environmental factors. Biochemistry 49 (2010) 4554-4563

29. Guédin, A., De Cian, A., Gros, J., Lacroix, L., Mergny, J.L.: Sequence effects in single-base loops for quadruplexes. Biochimie 90 (2008) 686-696

30. Guédin, A., Gros, J., Patrizia, A., Mergny, J.L.: How long is too long? Effects of loop size on G-quadruplex stability. Nucleic Acids Res. 38 (2010) 7858-7868

31. Lauhon, C.T., Szostak, J.W.: RNA aptamers that bind flavin and nicotinamide redox cofactors. J Am Chem Soc 117 (1995) 1246-1257

32. Joachimi, A., Benz, A., Hartig, J.S.: A comparison of DNA and RNA quadruplex structures and stabilities. Bioorg Med Chem 17 (2009) 6811-6815

33. Lorenz, R., Bernhart, S.H., Höner zu Siederdissen, C., Tafer, H., Flamm, C., Stadler, P.F., Hofacker, I.L.: ViennaRNA Package 2.0. Alg. Mol. Biol. 6 (2011) 26

34. Schuster, P., Fontana, W., Stadler, P.F., Hofacker, I.L.: From sequences to shapes and back: A case study in RNA secondary structures. Proc. Roy. Soc. Lond. B 255 (1994) 279-284

35. Flajolet, P., Sedgewick, R.: Analytic Combinatorics. Cambridge University Press, New York (2009)

36. Mathews, D.H., Disney, M.D., Childs, J.L., Schroeder, S.J., Zuker, M., Turner, D.H.: Incorporating chemical modification constraints into a dynamic programming algorithm for prediction ofRNA secondary structure. Proc. Natl. Acad. Sci. USA 101 (2004) 7287-7292

37. Zuker, M., Stiegler, P.: Optimal computer folding of large RNA sequences using thermodynamics and auxiliary information. Nucleic Acids Res. 9 (1981) 133-148

38. McCaskill, J.S.: The equilibrium partition function and base pair binding probabilities for RNA secondary structure. Biopolymers 29 (1990) 1105-1119

39. Bruccoleri, R.E., Heinrich, G.: An improved algorithm for nucleic acid secondary structure display. Computer Appl. Biosci. 4 (1988) 167-173

40. Do, C.B., Woods, D.A., Batzoglou, S.: CONTRAfold: RNA secondary structure prediction without physics-based models. Bioinformatics 22(14) (2006) e90-e98

41. Kumari, S., Bugaut, A., Huppert, J.L., Balasubramanian, S.: An RNA Gquadruplex in the 5'UTR of the NRAS proto-oncogene modulates translation. Nat. Chem. Biol. 3 (2007) 218-221

42. Hofacker, I.L., Fekete, M., Stadler, P.F.: Secondary structure prediction for aligned RNA sequences. J. Mol. Biol. 319 (2002) 1059-1066

43. Bernhart, S.H., Hofacker, I.L., Will, S., Gruber, A.R., Stadler, P.F.: RNAalifold: improved consensus structure prediction for RNA alignments. BMC Bioinformatics 9 (2008) 474

44. Hofacker, I.L., Priwitzer, B., Stadler, P.F.: Prediction of locally stable RNA secondary structures for genome-wide surveys. Bioinformatics 20 (2004) 191-198

45. Gros, J., Guédin, A., Mergny, J.L., Lacroix, L.: G-Quadruplex formation interferes with P1 helix formation in the RNA component of telomerase hTERC. ChemBioChem 9 (2008) 2075-2079

46. Ito, K., Go, S., Komiyama, M., Xu, Y.: Inhibition of translation by small RNAstabilized mRNA structures in human cells. J. Am. Chem. Soc. 133 (2011) 1915319159

47. Mückstein, U., Tafer, H., Hackermüller, J., Bernhard, S.B., Stadler, P.F., Hofacker, I.L.: Thermodynamics of RNA-RNA binding. Bioinformatics 22 (2006) 1177-1182 